\title{
Folliculin regulates cyclin D1 expression through cis-acting elements in the 3 ' untranslated region of cyclin D1 mRNA
}

\author{
AKIKO KAWAI, TOSHIYUKI KOBAYASHI and OKIO HINO \\ ${ }^{1}$ Department of Pathology and Oncology, Juntendo University School of Medicine; ${ }^{2}$ Department of Molecular Pathogenesis, \\ Juntendo University Graduate School of Medicine, Bunkyoku, Tokyo 113-8421, Japan
}

Received December 26, 2012; Accepted February 14, 2013

DOI: $10.3892 /$ ijo.2013.1862

\begin{abstract}
Birt-Hogg-Dubé syndrome (BHDS) is an autosomal dominantly inherited disease characterized by spontaneous pneumothorax, hair folliculomas and renal tumors. The responsible gene, $B H D$, is a tumor suppressor and encodes folliculin. Folliculin is an evolutionarily conserved protein $(\sim 67 \mathrm{kDa})$ with no apparent functional motif and its role has not yet been fully elucidated. In this study, we found that knockdown of $B H D$ increased the levels of cyclin D1 in HeLa cells. A reporter assay with the cyclin D1 gene (CCND1) promoter region indicated that this increase was not caused by activation of transcription through known cis-acting elements. We examined the possibility of post-transcriptional mechanism using reporter constructs containing fragments of the cyclin D1 3' untranslated region (3'UTR). Transfection of control cells with a construct carrying a medial $1.3 \mathrm{~kb}$ 3'UTR fragment resulted in a significant reduction in luciferase activity. This effect was largely prevented by knockdown of $B H D$. Our results suggest that the post-transcriptional regulation of the CCND1 expression by $B H D$ may be associated with microRNA(s) or RNA binding protein(s) that bind to the 3'UTR.
\end{abstract}

\section{Introduction}

Birt-Hogg-Dubé syndrome (BHDS) is an autosomal dominantly inherited disease characterized by spontaneous pneumothorax, hair folliculomas and renal tumors (1). The mutation responsible for BHDS has been shown by positional cloning to occur in the $B H D$ gene, which has tumor suppressor activity $(2,3)$. We previously investigated hereditary renal carcinoma in the Nihon rat model and identified a germline mutation in Bhd, the rat homolog of $B H D$ (4). Heterozygous Nihon mutant rats develop renal carcinomas that are mainly characterized by

Correspondence to: Professor Okio Hino or Dr Toshiyuki Kobayashi, Department of Pathology and Oncology, Juntendo University, School of Medicine, 2-1-1 Hongo, Bunkyoku, Tokyo 113-8421, Japan

E-mail: ohino@juntendo.ac.jp

E-mail: koba396@juntendo.ac.jp

Key words: BHD, folliculin, cyclin D1, 3' untranslated region clear-cell histology, while homozygotes for the mutation are embryonic lethal (5).

Human $B H D$ is located on chromosome 17p11.2 and encodes folliculin, an evolutionarily conserved protein $(\sim 67 \mathrm{kDa})$ that has no apparent functional motif (2). Although the function of folliculin has not been fully elucidated, two folliculin-interacting proteins, folliculin-interacting protein 1 (FNIP1) and its homolog folliculin-interacting protein 2 (FNIP2/FNIPL), have been identified (6-8). Baba et al demonstrated that 5' AMP-activated protein kinase (AMPK) interacts with FNIP1 and phosphorylates both FNIP1 and folliculin (6). They also reported the phosphorylation of folliculin is regulated directly or indirectly by mTOR. These findings suggest that folliculin is involved in energy and nutrient sensing through the AMPK and mTOR signaling pathways.

In the present study, we found that an increase in cyclin D1 protein levels was caused in HeLa cells by knockdown of $B H D$. Recently, it was reported that cyclin D1 protein levels were elevated in renal cell tumors of $B h d$ heterozygous knockout mice and renal cysts of kidney-specific Bhd knockout mice, and that the absence of folliculin resulted in an increase in CCND1 (cyclin D1 gene) mRNA levels in a $B H D$-deficient renal tumor cell line (UOK257) (9-11). CCND1 is an oncogenic cell cycle regulating gene and its aberrant activation may be implicated in the pathogenesis associated with $B H D$ deficiency (12). Here, we focus on the mechanism for the regulation by folliculin of cyclin D1 expression.

\section{Materials and methods}

Cell lines, culture conditions. HeLa cells were cultured in Dulbecco's modified Eagle's medium (DMEM; Sigma, St. Louis, MO, USA) containing 10\% fetal bovine serum (FBS) and antibiotics (penicillin/streptomycin). Treatment $(2 \mathrm{~h})$ with wortmannin (final concentration $100 \mathrm{nM}), \mathrm{SB} 203580(10 \mu \mathrm{M})$, $\mathrm{U} 0126(10 \mu \mathrm{M})$, rapamycin $(20 \mathrm{nM})$ or SP600125 $(20 \mu \mathrm{M})$ was performed using dimethylsulfoxide (DMSO) as the vehicle. Treatment with cycloheximide $(10 \mu \mathrm{g} / \mathrm{ml})$ was performed using ethanol as the vehicle.

Stable cell lines with an expression vector for folliculin were established from the NR32 (Bhd-deficient renal tumor cell line from the Nihon rat) (13). First, NR32 cells were transfected with the plasmid and stable cells were selected in DMEM containing 10\% FBS and G418 (final concentration $100 \mu \mathrm{g} / \mathrm{ml}$ ); 
clones were then selected after limiting dilution and cultured under the same conditions.

Plasmid construction. Rat full length Bhd cDNA was cloned into pcDNA3.1 vector (Invitrogen) (4). The CCND1 promoter region $(-1106$ to +159$)$ was amplified by PCR from HEK293 cell genomic DNA using the primers PHD1F1 (5'-CCGCTAGC CTCACGCTCACGAATTCAGT-3') and PHD1R1 (5'-CCAAG CTTATGGCTGGGGCTCTTCCT-3') and subcloned into pGL4.10 vector (Promega, Madison, WI, USA) after digestion with NheI and HindIII. Fragments of the 3' untranslated region (3'UTR) of human CCND1, 3'UTR1 (nt 1-1026) and 3'UTR2 (894-2253), were amplified from HEK293 cell genomic DNA using the following primers: CCNDIUTR1F (5'-GGTCTAGA AGCAGAACATGGACCCCAAG-3') and CCND1UTR1R (5'-GGTCTAGAGGACTGAAAGTGCTTGGAAA-3') for 3'UTR1; CCND1UTR2F (5'-CCTCTAGAACCTGTTTATG AGATGCTGG-3') and CCND1UTR2R (5'-CGTCTAGAGCC AAAGCAGGCAGAACCT-3') for 3'UTR2. Amplified products were subcloned into the $X b a I$ site (downstream of firefly luciferase coding region) of a vector consisting of pGL4.10 and the $C C N D 1$ promoter. A partial cDNA clone containing the distal $1.8 \mathrm{~kb} 3$ 'UTR of human CCNDI (HIBBN77) was obtained from American Type Culture Collection (Manassas, VA, USA). CCND1 3'UTR3 (2209-3171) was amplified from HIBBN77 using primers CCNDIUTR3F (5'-CCTCTAGACACAATAC CTCATGCTTCAC-3') and CCND1UTR3R (5'-TTTCTAGAC TTTCATGTTTGTCTTTTTG-3') and subcloned as 3'UTR1 and -2 . A construct containing the full length 3'UTR of CCND1 was generated from three partial constructs UTR1, -2 and -3 using PstI and NcoI sites. Full coding sequence of human HuR cDNA was amplified from HeLa cell cDNAs using the primers HuRF1 (5'-CGGAATTCGCCCGCATCCAGATTTTTGA-3') and HuRR1 (5'-GGCTCGAGTTTGTGGGACTTGTTGGT TTT-3') and subcloned into a modified pCAG-GS vector (pCAG-CFLAG) after digestion with EcoRI and XhoI to introduce a carboxy-terminal FLAG tag (14). A full length cDNA clone ( $\mathrm{pF} 1 \mathrm{~KB}$ 9904) for hnRNP L (heterogeneous nuclear ribonucleoprotein $\mathrm{L}$ ) was obtained from Promega and subcloned into $\mathrm{pCAG}-\mathrm{CFLAG}$ vector.

RNA interference (RNAi). Transfection of siRNA was performed with Lipofectamine RNAiMAX (Invitrogen, Carlsbad, CA, USA) at a final concentration of $25 \mathrm{nM}$. Sequences of siRNAs were described previously (8).

Antibodies. Antibodies against cyclin D1 (C-20 and H295), p27 (C-19), S6K (C-18), HuR (3A2) and hnRNP L (A-11) were purchased from Santa Cruz Biotechnology (Santa Cruz, CA, USA). Antibodies against phospho-p70 S6K (Thr389), phospho4E-BP1 (Thr37/46), 4E-BP and acetyl CoA carboxylase were obtained from Cell Signaling Technology (Danvers, MA, USA). Antibodies against histone H1 (AE4) and AUF1 were obtained from Millipore (Billerica, MA, USA). Anti- $\beta$-actin (AC15) was obtained from Sigma. The anti-folliculin (C1) antibody has been described previously (4).

Western blot analysis. Protein concentration was determined by the DC-protein assay (Bio-Rad, Hercules, CA, USA). Equal amounts of proteins were subjected to SDS-polyacrylamide gel electrophoresis and transferred to immobilon-P membranes (Millipore). The membranes were blocked in $1 \%$ skim milk in Tris-buffered saline containing $0.05 \%$ Tween-20 and then the appropriate antibodies were applied. Rabbit antibodies were detected using the Envision system (Dako, Glostrup, Denmark) as previously described (15). Mouse antibodies were detected using anti-mouse immunoglobulin and streptavidin-biotinylated horseradish peroxidase (GE Healthcare, Buckinghamshire, UK). ECL reagents (GE Healthcare) were used for detection.

RNA isolation, cDNA synthesis and quantitative RT-PCR $(q R T-P C R)$. Total RNA for mRNA analysis was extracted from cultured cells using FastPure RNA kit (Takara Bio, Ohtsu, Japan). cDNA was synthesized using SuperScript II Reverse Transcriptase with random primers (Invitrogen). qRT-PCR was performed using Power SYBR-Green PCR Master mix (Applied Biosystems, Life Technologies, Carlsbad,CA) and the following thermocycler conditions: $95^{\circ} \mathrm{C}$ for $10 \mathrm{~min}$, and 40 cycles of $95^{\circ} \mathrm{C}$ for $15 \mathrm{sec}$ and $60^{\circ} \mathrm{C}$ for $1 \mathrm{~min}$. All values were normalized against $\beta$-actin expression levels. Primer sequences were as follows: SBHCD1F1 (5'-TCGGTGTCCTACTTCAAATG-3') and SBHCD1R1 (5'-TTCTGTTCCTCGCAGACCTC-3') for CCND1; SBHCD3F1 (5'-GCCCTCTGTGCTACAGATT-3') and SBHCD3R1 (5'-CAGTCCACTTCAGTGCCAGT-3') for CCND3; (5'-CCATTTGCCATGGTTATAAGG-3') and SBHCER1 (5'-CTTTGCTCGGGCTTTGTCCA-3') for CCNE1; SBHVEGFAF1 (5'-CATGAACTTTCTGCTGTCTTGG-3') and SBHVEGFAR1 (5'-ATGATTCTGCCCTCCTCCTT-3') for VEGFA; SBHCDC25AF1 (5'-CAAGCGTGTCATTGTTG TGTT-3') and SBHCDC25AR1 (5'-CCCTTCAGGACATACA GCTCA-3') for $C D C 25 A$; SBHBAF1 (5'-CGCGAGAAGATG ACCCAGA-3') and SBHBAR1 (5'-GAGTCCATCACGATG CCAGT-3') for $\beta$-actin.

Reporter assay. After $24 \mathrm{~h}$ of siRNA treatment, HeLa cells were transfected with the various constructs using Fugene 6 (Roche, Mannheim, Germany). One microgram of each reporter construct was co-transfected with $20 \mathrm{ng}$ renilla luciferase expressing plasmid (pGL4.73, Promega). At $24 \mathrm{~h}$ after transfection, the cells were lysed for the analyses. PicaGene dual SeaPangy luminescence kit (Toyo B-net, Tokyo, Japan) was used to measure firefly and renilla luciferase activities.

miRNA analysis. Total RNA for microRNA analysis was isolated using the miRNeasy mini kit (Qiagen, Valencia, CA). cDNA was synthesized using the NCode VILO miRNA cDNA Synthesis kit (Invitrogen). qRT-PCR was performed using Express SYBR-GreenER qPCR SuperMixes and Two-Step qRT-PCR kits (Invitrogen) and the following thermocycler conditions: $50^{\circ} \mathrm{C}$ for $2 \mathrm{~min}, 95^{\circ} \mathrm{C}$ for $10 \mathrm{~min}$ and 40 cycles of $95^{\circ} \mathrm{C}$ for $15 \mathrm{sec}$ and $60^{\circ} \mathrm{C}$ for $1 \mathrm{~min}$. All values were normalized against U6 expression levels. The primer sequences for U6 and miR-16 amplification were described previously (16). Other primer sequences can be obtained from the NCode miRNA Database provided by Invitrogen (http://escience.invitrogen. com/ncode/).

Cell fractionation. Cytoplasmic and nuclear extracts from HeLa cells were obtained using NE-PER nuclear and cytoplasmic extraction reagent (Thermo Scientific, Waltham, MA, USA) 
A

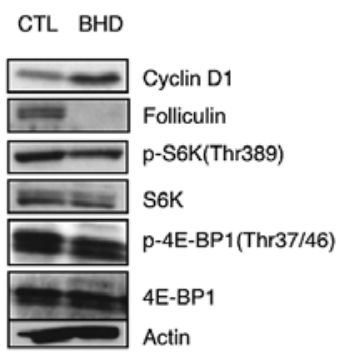

D

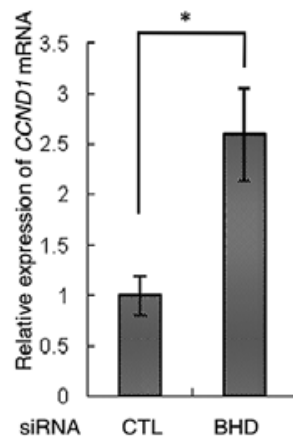

B

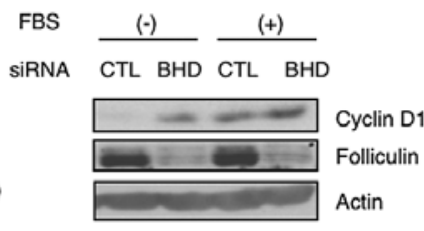

E

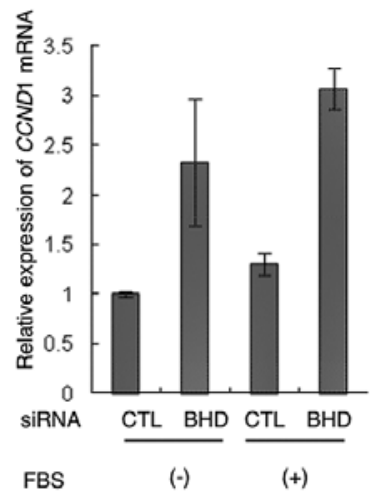

C

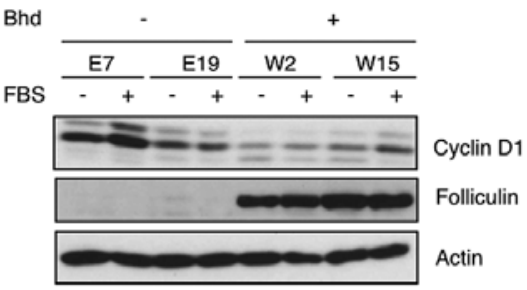

F

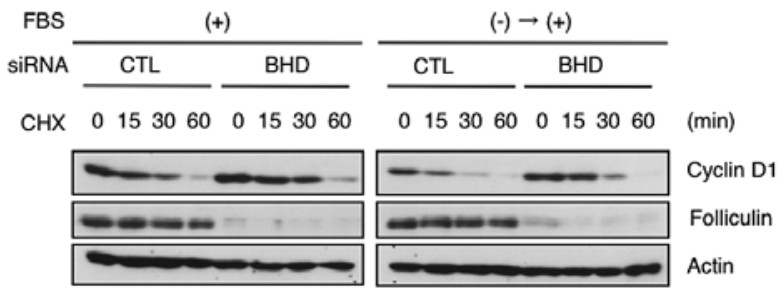

Figure 1. Regulation of cyclin D1 expression by folliculin. (A) Upregulation of cyclin D1 protein expression in HeLa cells by $B H D$ knockdown. HeLa cells were transfected with control (CTL) or BHD siRNA (BHD). Lysates were analyzed by western blot analysis with the indicated antibodies. $\beta$-actin was used as the control. (B) Increased expression of cyclin D1 protein in BHD knockdown cells during serum starvation. HeLa cells were transfected as in (A). During the final $24 \mathrm{~h}$ of culture, the cells were starved of serum and then cultured in the presence (+) or absence (-) of $10 \%$ serum (FBS) for $2 \mathrm{~h}$. Western blot analysis was performed with the indicated antibodies. (C) Expression of cyclin D1 in Bhd-deficient (E7, E19) and Bhd-restored (W2, W15) cells. FBS (-), serum starvation for 24 h; (+), with serum for $24 \mathrm{~h}$. Lysates were analyzed by western blot analysis with the indicated antibodies. (D) Relative change in $C C N D 1$ mRNA levels induced by $B H D$ knockdown. CCND1 mRNA expression in control HeLa (CTL) and BHD knockdown cells (BHD) was analyzed by qRT-PCR. Error bars represent SD; ${ }^{*}<0.05$. Three independent experiments were performed in triplicate. (E) qRT-PCR of the relative expression levels of $C$ CND1 under serum starvation conditions. As in (B), during the last $24 \mathrm{~h}$ of the siRNA treatment, the cells were starved of serum and then grown for $2 \mathrm{~h}$ in the presence (+) or absence (-) of $10 \%$ serum (FBS). Error bars represent SD. Three independent experiments were performed in triplicate. (F) Stability of cyclin D1 protein. HeLa cells were transfected with control (CTL) or BHD siRNA (BHD) and cultured under different culture conditions: FBS(+), with serum; FBS(-) $\rightarrow(+)$, cells were arrested by serum starvation for 24 h, and then grown for $2 \mathrm{~h}$ in DMEM containing 10\% FBS. Cycloheximide (CHX) was added for the indicated time; lysates were analyzed by western blot analysis with the indicated antibodies.

with protease inhibitors (aprotinin, leupeptin, pepstatin A) following the manufacturer's instructions.

\section{Results}

Increase in cyclin D1 levels by BHD knockdown. siRNA mediated suppression of $B H D$ expression in HeLa cells caused an increase in expression of cyclin D1 protein (Fig. 1A).Upregulation of cyclin D1 levels was observed in BHD suppressed cells even under serum starvation conditions (Fig. 1B). We examined cyclin D1 protein levels in rat renal tumor cell lines that had been stably transduced with an expression vector for folliculin or an empty vector as a control; the cell lines were derived from NR32, a Bhd deficient renal tumor cell line from the Nihon rat. Cyclin D1 levels in the cell lines with restoration of $B h d$ activity (W2 and W15 in Fig. 1C) were lower than in Bhd deficient cells (E7 and E19) in both serum starvation and serum added conditions. These results suggest that the downstream pathway of folliculin negatively regulates cyclin D1 expression.

To determine whether the induced increase in cyclin D1 levels after $B H D$ knockdown was a result of upregulation of mRNA expression, we performed qRT-PCR. This analysis showed that CCND1 mRNA levels in BHD knockdown cells were also higher than those in control cells (Fig. 1D and E). We also examined the half-life of cyclin D1 using cycloheximide to ascertain whether cyclin D1 turnover was downregulated by $B H D$ knockdown in HeLa cells; no significant difference was present (Fig. 1F). These results suggest that folliculin regulates cyclin D1 expression at transcriptional or posttranscriptional level but not via protein turnover.

BHD knockdown does not stimulate CCND1 promoter activity. To determine whether folliculin regulates the transcription of $C C N D 1$, we carried out a reporter analysis by transfecting HeLa cells with a luciferase expressing vector carrying the $C C N D 1$ promoter region $(-1106$ to +159$)(15)$. Although the $C C N D 1$ mRNA levels increased, luciferase activity was not elevated but rather suppressed in BHD knockdown cells (Fig. 2A). Therefore, we concluded that folliculin-mediated control of the level of CCNDI mRNA is likely not to be via transcriptional mechanisms through known cis-acting elements.

Regulation of CCND1 mRNA level through 3'UTR by folliculin. Next, we tested whether the expression of CCNDl was regu- 
A

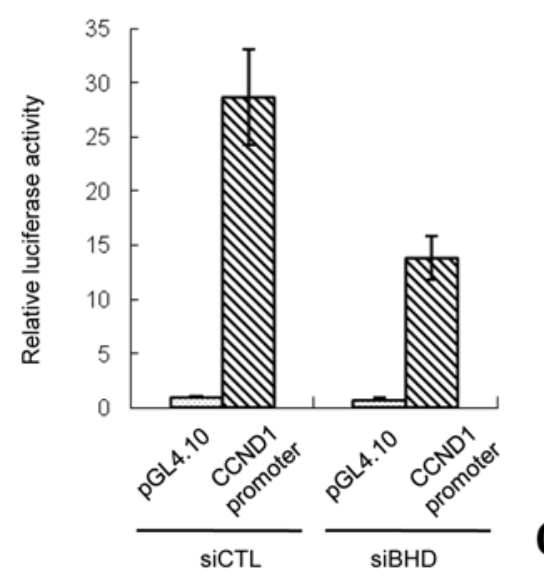

B
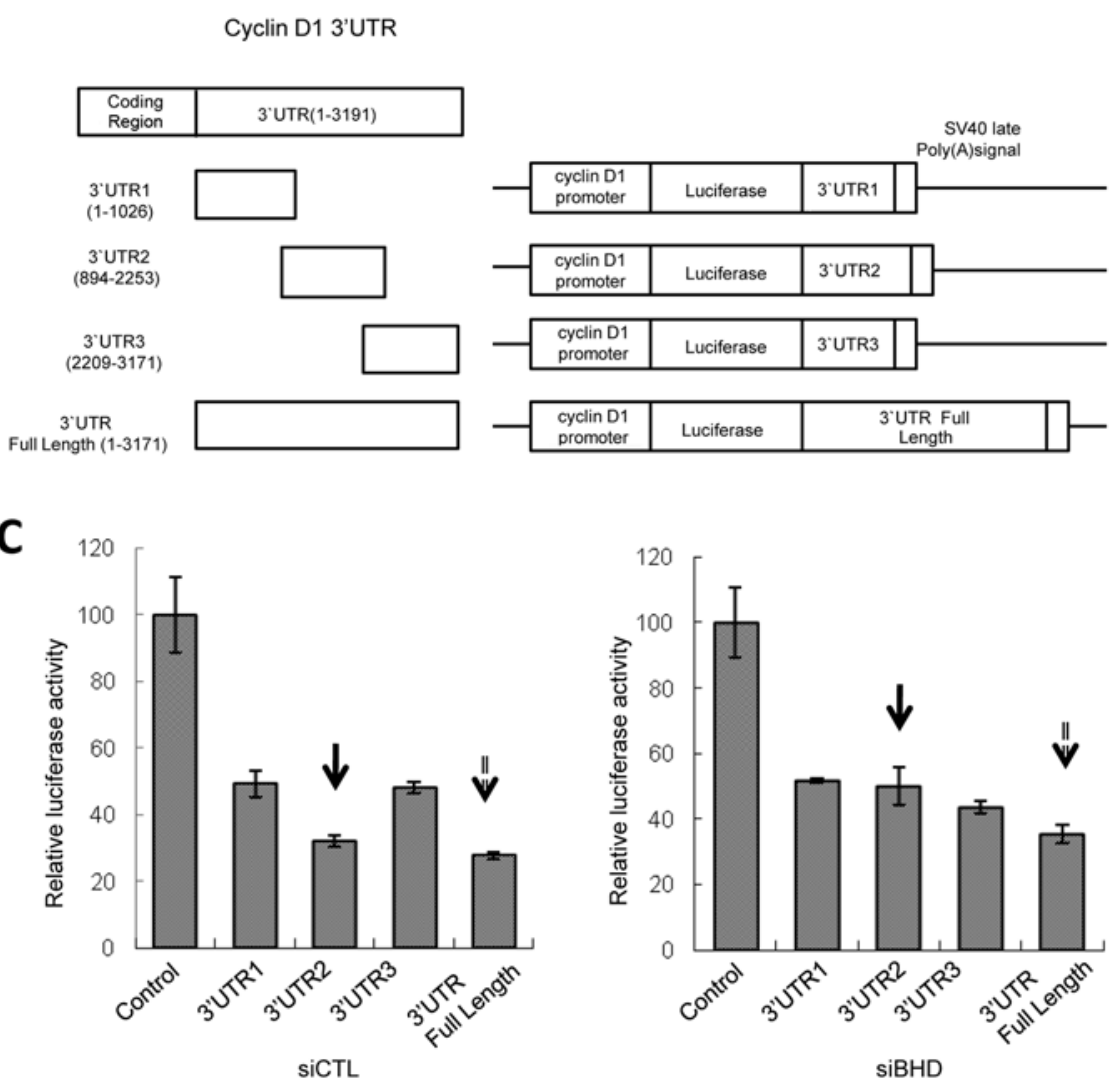

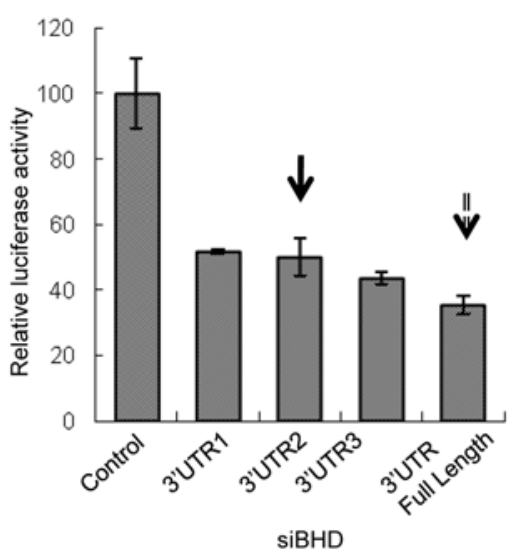

Figure 2. Reporter analyses of $C C N D 1$ promoter and 3'UTR of mRNA. (A) Transcriptional activity of the $C C D N 1$ promoter following $B H D$ knockdown. HeLa cells were transfected with control (siCTL) or $B H D$ siRNA (siBHD), then transfected with a luciferase expression plasmid carrying the $C C N D 1$ promoter $(-1106$ to +159$)$ or a pGL4.10 control vector. Lysates were analyzed for luciferase fluorescence to determine the transcriptional activity of the $C C N D 1$ promoter. Three independent experiments were performed in triplicate. (B) Schematic structures of the reporter constructs containing the fragments of the cyclin D1 3'UTR. $C C N D 1$ 3'UTR (3.1 kb) was divided into three overlapping regions and inserted into a luciferase expression vector. (C) Effects of $C C D N 1$ 3'UTR fragments on reporter expression. HeLa cells were transfected with control (siCTL) or BHD siRNA (siBHD), then transfected with reporter constructs carrying the $C C N D 1$ 3'UTR fragments or with a control plasmid. Lysates were analyzed for luciferase fluorescence. Error bars represent SD. Three independent experiments were performed in triplicate.

lated by 3'UTR of mRNA. Three constructs carrying different 3'UTR fragments (3'UTR1-3) of CCND1 (Fig. 2B) exhibited inhibition of reporter activity, possibly due to the existence of negative regulatory elements. Knockdown of $B H D$ showed no effect on reporter activity of the constructs containing 3'UTR1 or 3'UTR3. However, for the third construct, 3'UTR2, BHD knockdown increased reporter activity (Fig. 2C, arrows with solid line) and this phenomenon was not cancelled by rapamycin treatment (data not shown). Full length $C C N D 1$ 3'UTR also gave similar results, although to a lesser extent than 3'UTR2 (Fig. 2C, arrows with dotted line). We also tested the effects of 3'UTR2 inserted in reverse orientation or upstream of the promoter region. Neither of these constructs elevated luciferase activity in $B H D$ knockdown cells compared to control cells, indicating that the function of 3'UTR2 is position and orientation dependent (data not shown). These results imply that the 3'UTR2 sequence in the CCND1 mRNA influences the level of expression level and that the regulation of CCNDI by folliculin may be associated with microRNA(s) or RNA binding protein(s) (RBPs) that binds to the 3'UTR2.

CCND1 3'UTR-related microRNAs and BHD knockdown. It was reported previously that the $3^{\prime} \mathrm{UTR}$ of $C C N D 1$ is a
Table I. List of microRNAs predicted to target cyclin D1 by TargetScan.

miR-15a, miR-15b, miR-16, miR 17, miR-19a, miR-19b, miR-20a, miR-20b, miR-34a, miR-93, miR-106a, miR-106b, miR-155, miR-195, miR-302a, miR-302b, miR-302c, miR-302d, miR-424, miR-497, miR-503, miR-519d

target of several microRNAs or RBPs and that these transacting factors regulate CCNDI mRNA stability and/or protein expression $(16,18,19)$. We hypothesized that BHD knockdown alters the regulatory pathway that controls microRNA or RBP binding to CCND1 3'UTR. We searched for putative microRNA binding sites on CCND1 3'UTR using the publicly accessible database TargetScan (http://www.targetscan.org) and evaluated the results by comparison with those described in a previous report (Fig. 3) (16). Several of the potential microRNA targets were found to be conserved among vertebrates. We carried out a qRT-PCR on 22 candidate microRNAs (Table I), but did not identify any clear change in their expression levels following $B H D$ knockdown (Fig. 4A, 


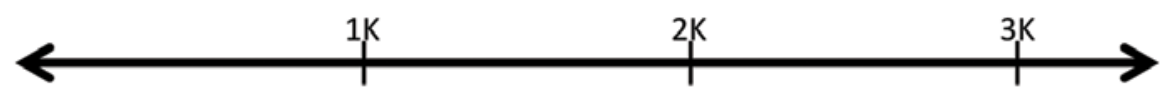

Human CCND1 NM_053056 3'UTR length: 3191

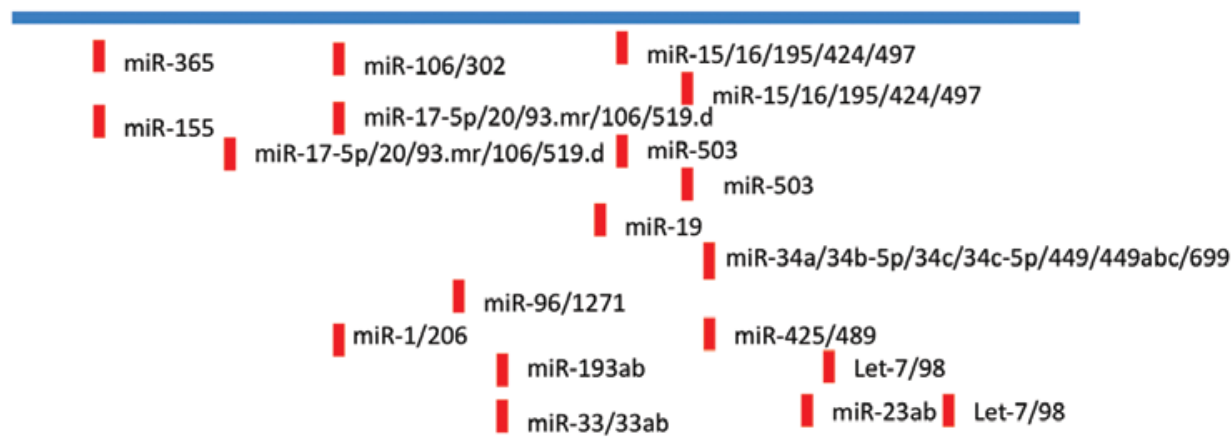

\section{I: Conserved sites for miRNA famillies broadly concerved among vertebrates.}

Figure 3. Human $C C N D 1$ 3'UTR and possible miRNA target sites predicted by TargetScan.

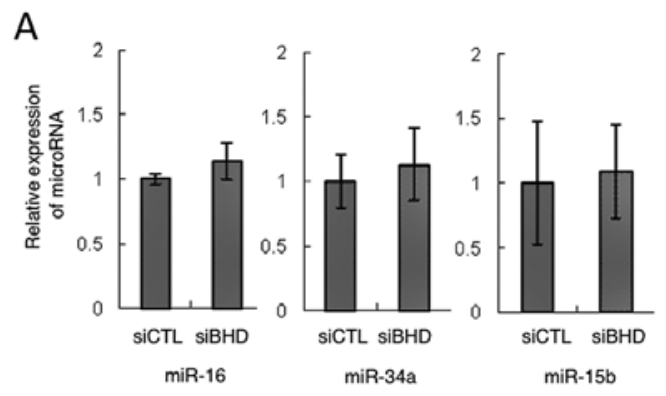

C

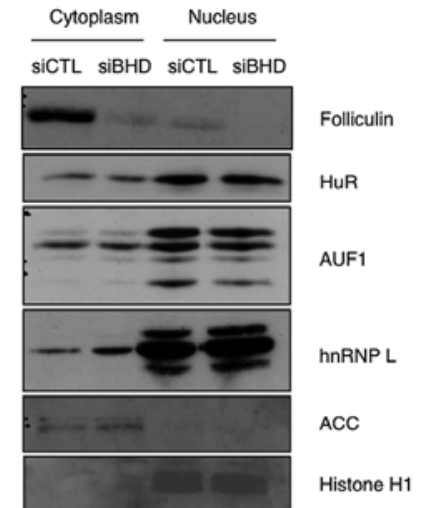

B

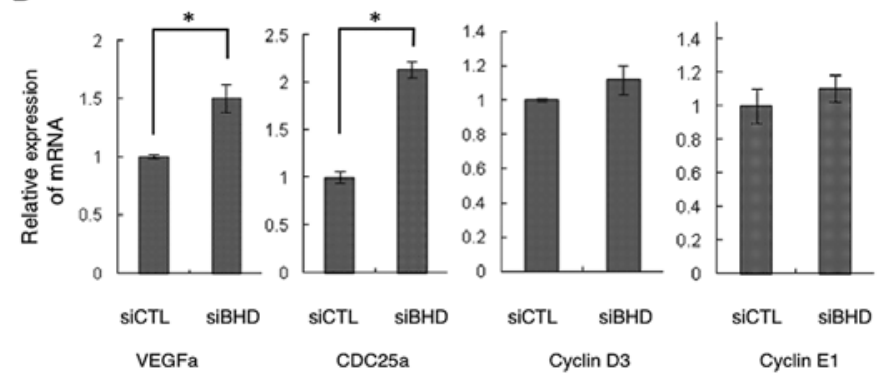

D

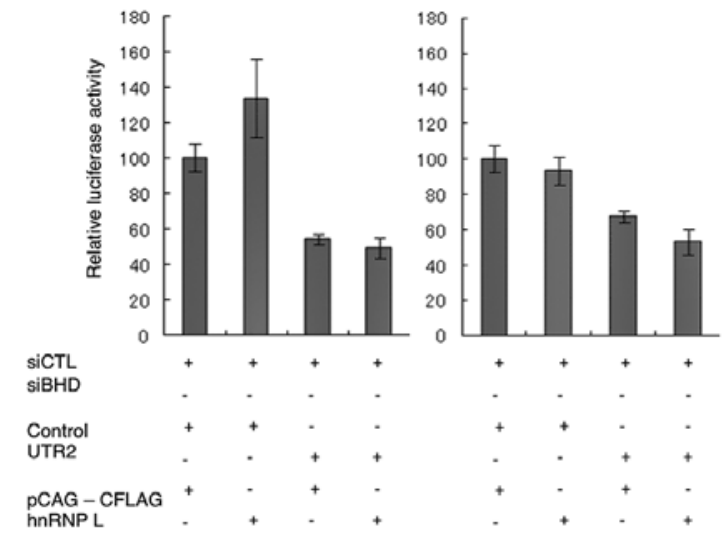

Figure 4. Analyses for microRNAs and 3'UTR binding proteins in BHD knockdown cells. HeLa cells were treated with control (siCTL) or BHD siRNA (siBHD). (A and B) qRT-PCR analyses for (A) microRNAs that target cyclin D1 mRNA and (B) for genes downstream of miR-16. Error bars represent SD. Three independent experiments were performed in triplicate. ${ }^{*} \mathrm{P}<0.05$. (C) Cell fractionation analysis for 3 'UTR binding proteins. Cytoplasmic and nuclear fractions of HeLa cell extracts were analyzed by western blot analysis for HuR, AUF1 and hnRNP L. Acetyl CoA carboxylase (ACC) and histone H1 were analyzed as marker proteins for cytoplasmic and nuclear fractions, respectively. (D) Reporter assay of co-expression of hnRNP L. siRNA-treated cells were co-transfected with a reporter construct (control, promoter only; UTR2, promoter with CCND1 3'UTR2) and hnRNP L-expression plasmid or pCAG-CFLAG (empty). Lysates were anayzed for luciferase activity. Error bars represent SD. Two independent experiments were performed in triplicate.

and data not shown). We also investigated the mRNA levels of miR-16 target genes $(16,19-21)$. miR-16 is believed to be a major microRNA that binds to the CCNDI 3'UTR. The analysis showed that $B H D$ knockdown increased the relative level of expression of $V E G F A, C D C 25 A$ mRNA, but not of $C C N D 3$ or $C C N E 1$ (Fig. 4B). From these observations, we 
A

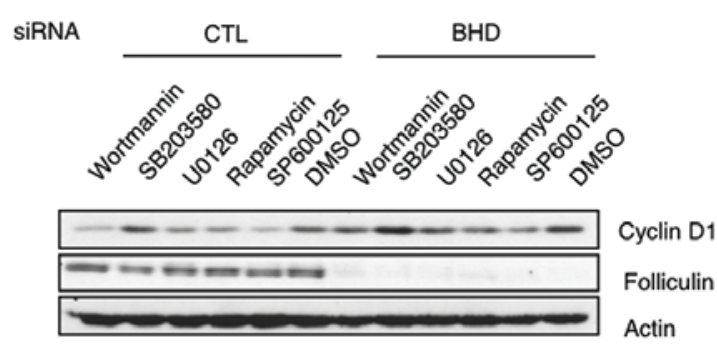

B

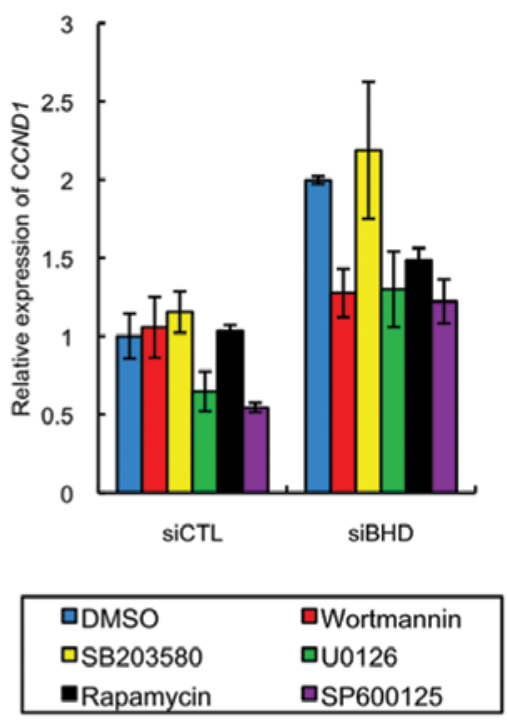

Figure 5. Changes in cyclin D1 protein and mRNA levels induced by inhibitors. (A) HeLa cells were transfected with control (CTL) or BHD siRNA (BHD). During the last $24 \mathrm{~h}$, cells were serum-starved and then treated with wortmannin (PI3K inhibitor), SB203580 (p38 inhibitor), U0126 (MEK1/2 inhibitor), rapamycin (mTOR inhibitor), SP600125 (JNK inhibitor) or DMSO for $2 \mathrm{~h}$. Lysates were analyzed by western blot analysis using the indicated antibodies. (B) Relative expression levels of CCND1 mRNA were compared between control cells (siCTL) and BHD knockdown cells (siBHD) treated with the indicated inhibitors by qRT-PCR. Error bars represent SD. Three independent experiments were performed in triplicate.

conclude that the microRNAs listed here do not play a major role in cyclin D1 regulation by folliculin.

RNA binding proteins and BHD knockdown. We analyzed three RBPs, HuR, AUF1 and hnRNP L, that have previously been reported to bind to 3'UTR and to control mRNA stability. HuR and AUF1 have been identified as binding to the AU rich element (ARE) on the CCND1 3'UTR. HuR stabilizes CCND1 mRNA, whereas AUF1 causes its destabilization (18). hnRNPL has been reported to bind to the CA rich element of VEGFA and to increase mRNA stability (22). First we performed a cell fractionation analysis and found that cytoplasmic hnRNP L levels were increased by $B H D$ knockdown (Fig. 4C), whereas those of HuR and AUF1 were unchanged in both the cytoplasm and nucleus. To determine whether these RBPs could bind CCNDI 3'UTR and regulate mRNA expression in a folliculin-dependent manner, a reporter assay using constructs expressing hnRNP L and HuR was performed. We found that luciferase activities from the 3'UTR2-containing reporter were not elevated following co-transfection of hnRNP L or HuR, regardless of the folliculin status (Fig. 4D and data not shown). Accordingly, although possible regulation of hnRNP L localization was suggested, the folliculin-dependent association between 3'UTR2 and RBPs could not be identified in our experiments.

BHD knockdown elevates rapamycin sensitivity of CCNDI $m R N A$ expression. To explore the signaling pathway modulated after $B H D$ knockdown, we investigated the effects of several signal inhibitors on cyclin D1 expression. This analysis showed that 2-h treatment of wortmannin, U0126, rapamycin or SP600125 inhibited cyclin D1 protein levels in control and BHD knockdown cells, whereas SB203580 was ineffective in both cell types (Fig. 5A). Each of the positive inhibitors had a similar effect in control and $B H D$ knockdown cells relative to the vehicle control. Interestingly, the relative levels of $C C N D 1$ mRNA in drug treated $B H D$ knockdown cells differed from that in control cells (Fig. 5B). Treatment with wortmannin or rapamycin significantly decreased the relative levels of CCND1 mRNA in a $B H D$ knockdown-specific manner (Fig. 5B). These results suggest that the regulatory mechanism for $C C N D 1$ mRNA expression was more sensitive to inhibition of the mTORC1 pathway in $B H D$ knockdown cells.

\section{Discussion}

Although $B H D$ is regarded as a tumor suppressor gene, its function is not clearly understood and its contribution to cell cycle regulation, including the suppression of cyclin D1, has yet to be determined. In this study, we found an increase in cyclin D1 protein and mRNA levels in BHD knockdown cells. Based on the results of our experiments, we suggest that the increase in CCND1 mRNA and protein levels induced by BHD suppression is mediated, at least in part, by a 3'UTR (3'UTR2)-related mechanism.

The $C C N D 1$ promoter region $(-1106$ to +159$)$ used in this study was reported to contain core positive regulatory elements that respond to cell cycle promoting signals (17). Although we did not identify any element in the $C C N D 1$ promoter region that responded to $B H D$ downregulation, the existence of such cis-acting elements in other regions of the CCNDI gene cannot be excluded.

One of the mechanisms for regulation of mRNA levels is microRNA-mediated degradation (23). There are a number of microRNA binding sites in the CCND1 3'UTR2 and many 
of these are conserved between humans and rat (14). For example, miR-16 has been implicated in the regulation of cell cycle related genes including cyclin D1 (16,19-21). The increase in CCND1 mRNA levels after knockdown of $B H D$ could be explained as the consequence of downregulation by a microRNA-mediated mechanism. To date, we have not identified any expression change in microRNAs nor of miR-16 target genes in $B H D$ knockdown cells. However, as there are additional microRNA binding sites to those analyzed in 3'UTR2, the potential contribution of other microRNAs remains to be determined. Possibly, comprehensive microRNA analysis will identify candidates for 3'UTR2-associated miRNAs downstream of folliculin.

The CCND1 3'UTR has been reported to have seven AREs composed of an AUUUA pentamer; six of these seven motifs are present in 3'UTR2 (24). Many RBPs have been found to selectively recognize and bind to the ARE of mRNAs and to modulate the translation and/or stability of the mRNAs (25). We tested the effects of three RBPs (HuR, AUF1, hnRNP L) on 3'UTR2-mediated regulation, but did not find a relationship between these RBPs and folliculin. Other RBPs may bind CCND1 mRNA 3'UTR2 to regulate folliculin-mediated mRNA translation/stability. Interestingly, cytoplasmic hnRNP L levels were increased by $B H D$ knockdown in our study. hnRNP L has been reported to bind to $\mathrm{CA}$ repeats/CA rich elements in the 3'UTR of VEGFA, BCL-2 and GLUT1, and to increase the stability of their mRNAs $(22,26,27)$. Notably, the CCNDI 3'UTR2 also has CA repeats. Jafarifar et al reported that hypoxia induces translocation of nuclear hnRNP L to the cytoplasm, resulting in a marked increase in hnRNP L binding to VEGFA mRNA and in the stability of VEGFA mRNA (22). Although our reporter analysis did not detect any specific effects, it is possible that in CCND1 3'UTRs other than 3'UTR2, some cooperative elements are required for the efficient binding and regulation by hnRNP L. Singh et al (28) suggested that folliculin may be a component of an RBP complex on the basis of observation that the Drosophila folliculin protein binds to RBP9, a homologue of human Hu protein, and shows weak homology to Pumilio, another RBP (29). Thus, the possibility of direct binding and regulation of CCND1 mRNA 3'UTR2 by a folliculin-RBP complex still remains.

We performed a search for signaling molecules involved in the upregulation of cyclin D1 expression using various signal inhibitors. This analysis showed that rapamycin and wortmannin suppressed CCND1 mRNA levels in BHD knockdown cells but not in control cells. These results suggest that the mTORC1 pathway may contribute to upregulation of CCND1 expression caused by $B H D$ knockdown. Rapamycin has been reported to affect $C C N D 1 \mathrm{mRNA}$ and protein stability and thereby decrease levels of cyclin D1 protein (30). Recent studies have also reported that rapamycin regulates $C C N D 1$ transcription and mRNA stability in an AKT-dependent manner; under conditions of relatively quiescent AKT activity, treatment of cells with rapamycin results in the upregulation of CCNDI transcription and mRNA stability, whereas in cells containing active AKT, rapamycin these changes are repressed $(31,32)$. This phenomenon may be related to the rapamycin sensitivity of $B H D$ knockdown cells found in this study. We demonstrated that S6K1 phosphorylation and total mTOR levels are decreased by $B H D$ knockdown in HeLa cells, and this also may lead to an increased sensitivity to the effects of rapamycin and wortmannin (8). Cyclin D1 protein and mRNA levels were decreased by rapamycin treatment in $B H D$ knockdown cells, but this treatment did not affect the upregulated reporter activity by 3'UTR2 in these cells. Thus, although mTORC1 signaling is required, it may be not a direct cause of the CCNDI mRNA increase caused by $B H D$ downregulation.

In recent studies, it has been reported that cyclin D1 protein levels were elevated in renal cell tumors of $B h d$ heterozygous knockout mice and the absence of folliculin resulted in an increase in CCND1 mRNA levels in UOK257 cells $(10,11)$. Analysis of the folliculin-mediated mechanism regulating cyclin D1 expression may provide important clues to understanding the pathogenesis of BHDS and other BHD mutation-associated diseases.

\section{Acknowledgements}

We thank Dr K. Kajino, Dr S. Matsuoka, Dr D. Zhang, Dr Y. Ito, Dr H. Kawano, Dr X. Piao, Dr L. Wang, Mr. M. Abe and Mr. T. Takagaki in the Department of Pathology and Oncology for helpful discussion and technical supports. We also thank Mrs. T. Ikegami in the Division of Molecular and Biochemical Research, Biochemical Research Center (Juntendo University Graduate School of Medicine) for expert support. We appreciate Dr K. Okimoto, Dr I. Matsumoto and Dr M. Kouchi (Dainippon-Sumitomo Pharma) for providing NR32. This study was supported by the grants from the Japan Society for the Promotion of Science (JSPS), from the Ministry of Education, Culture, Sports and Technology of Japan, and from the Ministry of Health, Labour and Welfare of Japan.

\section{References}

1. Birt AR, Hogg GR and Dubé WJ: Hereditary multiple fibrofolliculomas with trichodiscomas and acrochordons. Arch Dermatol 113: 1674-1677, 1977.

2. Nickerson ML, Warren MB, Toro JR, et al: Mutations in a novel gene lead to kidney tumors, lung wall defects, and benign tumors of the hair follicle in patients with the Birt-Hogg-Dubé syndrome. Cancer Cell 2: 157-164, 2002.

3. Vocke CD, Yang Y, Palvovich CP, et al: High frequency of somatic frameshift BHD mutations in Birt-Hogg-Dubéassociated renal tumors. J Natl Cancer Inst 97: 931-935, 2005.

4. Okimoto K, Sakurai J, Kobayashi T, et al: A germ-line insertion in the Birt-Hogg-Dubé (BHD) gene gives rise to the Nihon rat model of inherited renal cancer. Proc Natl Acad Sci USA 101: 2023-2027, 2004.

5. Okimoto K, Kouchi M, Matsumoto I, Sakurai J, Kobayashi T and Hino O: Natural history of the Nihon rat model of BHD. Curr Mol Med 4: 887-893, 2004.

6. Baba M, Hong SB, Sharma N, et al: Folliculin encoded by the BHD gene interacts with a binding protein, FNIP1, and AMPK, and is involved in AMPK and mTOR signaling. Proc Natl Acad Sci USA 103: 15552-15557, 2006.

7. Hasumi H, Baba M, Hong SB, et al: Identification and characterization of a novel folliculin-interacting protein FNIP2. Gene 415: 60-67, 2008.

8. Takagi Y, Kobayashi T, Shiono M, et al: Interaction of folliculin (Birt-Hogg-Dubé product) with a novel Fnip1-like (FnipL/Fnip2) protein. Oncogene 27: 5339-5347, 2008.

9. Baba M, Furihata M, Hong SB, et al: Kidney-targeted Birt-Hogg-Dubé gene inactivation in a mouse model: Erk $1 / 2$ and Akt-mTOR activation, cell hyperproloferation, and polycystic kidneys. J Natl Cancer Inst 100: 140-154, 2008.

10. Hasumi Y, Baba M, Ajima R, et al: Homozygous loss of BHD causes early embryonic lethality and kidney tumor development with activation of mTORC1 and mTORC2. Proc Natl Acad Sci USA 106: 18722-18727, 2009. 
11. Preston RS, Philip A, Classens T, et al: Absence of Birt-Hogg-Dubé gene product is associated with increased hypoxia-inducible factor transcriptional activity and a loss of metabolic flexibility. Oncogene 30: 1159-1173, 2010.

12. Sherr CJ and Roberts JM: CDK inhibitors: positive and negative regulators of G1-phase progression Genes Dev 13: 1501-1512, 1999.

13. Matsumoto I, Kouchi M, Okimoto K, et al: Establishment and characterization of renal carcinoma cell lines from a Bhd gene mutant (Nihon) rat. Tumour Biol 30: 249-256, 2009.

14. Niwa H, Yamamura K and Miyazaki J: Efficient selection for high-expression transfectants with a novel eukaryotic vector. Gene 108: 193-199, 1991.

15. Fukuda T, Tani Y, Kobayashi T, Hirayama Y and Hino O: A new western blotting method using polymer immunocomplexes: detection of Tsc1 and Tsc2 expression in various cultured cell lines. Anal Biochem 285: 274-276, 2000.

16. Chen RW, Bemis LT, Amato CM, et al: Truncation in CCND1 mRNA alters miR-16-1 regulation in mantle cell lymphoma. Blood 112: 822-829, 2008.

17. Tetsu $\mathrm{O}$ and McCormick $\mathrm{F}$ : $\beta$-catenin regulates expression of cyclin D1 in colon carcinoma cells. Nature 398: 422-426, 1999.

18. Lal A, Mazan-Mamczarz K, Kawai T, Yang X, Martindale JL and Gorospe M: Concurrent versus individual binding of HuR and AUF1 to common labile target mRNAs. EMBO J 23: 3092-3102, 2004.

19. Liu Q, Fu H, Sun F, et al: miR-16 family induces cell cycle arrest by regulating multiple cell cycle genes. Nucleic Acids Res 36: 5391-5404, 2008.

20. Hua Z, Lv Q, Ye W, et al: miRNA-directed regulation of VEGF and other angiogenic factors under hypoxia. PLoS One 1: e116, 2006.

21. Pothof J, Verkaik NS, van Ijcken W, et al: MicroRNA-mediated gene silencing modulates the UV-induced DNA damage response. EMBO J 28: 2090-2099, 2009.

22. Jafarifar F, Yao P, Eswarappa SM and Fox PL: Repression of VEGFA by CA-rich element-binding microRNAs is modulated by hnRNP L. EMBO J 30: 1324-1334, 2011.
23. Guo H, Ingolia NT, Weissman JS and Bartel DP: Mammalian microRNAs predominantly act to decrease target mRNA levels. Nature 466: 835-840, 2010.

24. Deshpande A, Pastore A, Deshpande AJ, et al: 3'UTR mediated regulation of cyclin D1 proto-oncogene. Cell Cycle 32: 3592-3600, 2009.

25. Barrreau C, Paillard L and Osborne HB: AU-rich elements and associated factors: are there unifying principles? Nucleic Acids Res 33: 7138-7150, 2005.

26. Hamilton BJ, Nichols RC, Tsukamoto H, Boado RJ, Pardridge WM and Rigby WF: hnRNP A2 and hnRNP L bind the 3 'UTR of glucose transporter 1 mRNA and exist as a complex in vivo. Biochem Biophys Res Commun 261: 646-651, 1999.

27. Lee DH, Lim MH, Youn DY, Jung SE, Ahn YS, Tsujimoto Y and Lee JH: hnRNP L binds to CA repeats in the 3'UTR of bcl-2 mRNA. Biochem Biophys Res Commun 382: 583-587, 2009.

28. Singh SR, Zhen W, Wang H, et al: The Drosophila homolog of the human tumor supressor gene BHD interacts with the JAK-STAT and Dpp signaling pathways in regulating male germline stem cell maintenance. Oncogene 25: 5933-5941, 2006.

29. Park SJ, Yang ES, Kim-Ha J and Kim YJ: Down regulation of extramacrochaetae mRNA by drosophila neural RNA binding protein $\mathrm{Rbp} 9$ which is homologous to human $\mathrm{Hu}$ proteins. Nucleic Acids Res 26: 2989-2994, 1998.

30. Hashemolhosseini S, Nagamine Y, Morley S, Desrivières S, Mercep L and Ferrari S: Rapamycin inhibition of G1 to S transition is mediated by effect on cyclin D1 mRNA and protein stability. J Biol Chem 273: 14424-14429, 1998

31. Manderosian M, Sharma A, Funk AP, Vartanian R, Mastri J, Jo OD and Gera JF: Tristetraprolin regulates cyclin D1 and c-Myc mRNA stability in response to rapamycin in an Akt-dependant manner via p38 MAPK signaling. Oncogene 25: 6277-6290, 2006

32. Vartanian R, Mastri J, Martin J, et al: AP-1 regulates cyclin D1 and c-MYC transcription in an AKT-dependent mannner in response to mTOR inhibition: role of AIP4/Itch-mediated JUNB degradation. Mol Cancer Res 9: 115-130, 2011. 